\title{
Acute and Long-Term Treatments with an Herbal Formula V-Vital Capsule Increase Exercise Endurance Capacity in Weight-Loaded Swimming Mice
}

\author{
Pou Kuan Leong1, Hoi Yan Leung1, Wing Man Chan'1, Ji Hang Chen¹, Hoi Shan Wong1, \\ Chung Wah Ma², Shi Yu Zou², Kam Ming Ko' ${ }^{*}$ \\ ${ }^{1}$ Division of Life Science, The Hong Kong University of Science \& Technology, Hong Kong SAR, China \\ ${ }^{2}$ Infinitus (China) Company Ltd., Guangzhou, China \\ Email: "bcrko@ust.hk
}

Received 22 June 2014; revised 19 July 2014; accepted 31 July 2014

Copyright (C) 2014 by authors and Scientific Research Publishing Inc. This work is licensed under the Creative Commons Attribution International License (CC BY). http://creativecommons.org/licenses/by/4.0/

(c) ()

\section{Abstract}

Fatigue is a self-limiting response arising from physical and/or mental weariness, with a consequent personal and economic morbidity on work performance and social relationships. Anti-fatigue intervention is therefore urgently sought. "Qi-invigorating" Chinese tonic herbs, which can improve the energy status in the body according to the theory of traditional Chinese medicine, may produce beneficial effects in fatigue individuals. The herbal formula V-Vital capsule (VVC), which comprises 3 "Qi-invigorating" herbs, namely the root of Rhodiola rosea, Eleutherococcus senticosus and Panax quinquefolium, may produce anti-fatigue effect. In the present study, we investigated the effect of acute/long-term VVC treatment (acute: $0.75,0.2$ and $3.75 \mathrm{~kg} /$ day $\times 1$ dose; long-term: 0.075 and $0.25 \mathrm{~g} / \mathrm{kg} /$ day $\times 14$ doses) on weight-loaded swimming female ICR mice. The weight-loaded swimming time until exhaustion, indicative of exercise endurance capacity, was recorded. Plasma levels of glucose, non-esterified fatty acid (NEFA), lactate and reactive oxygen metabolites (ROM) were measured in the exhausted mice. Glycogen levels in skeletal muscle and liver tissues were also measured. Mitochondrial function status [such as adenine nucleotide translocase (ANT) activity and coupling efficiency] was assayed. Results showed that acute VVC treatment increased the exercise endurance capacity in weight-loaded swimming mice. The ability of acute VVC treatment to enhance the exercise endurance was associated with increases in plasma glucose levels as well as glycogen levels in skeletal muscles and liver tissues, presumably due to the utilization of plasma lactate for gluconeogenesis and/or glycogen synthesis in the liver. While acute VVC treatment reduced the plasma ROM level in weight-loaded swimming mice, it increased the ANT activity. In this regard, the enhancement in exercise endurance afforded by acute ${ }^{*}$ Corresponding author. 
VVC treatment might be due to an increase in the glucose supply to the skeletal muscle, the amelioration of systemic oxidative stress and the improvement in mitochondrial function of skeletal muscle. Consistent with the results obtained in acute VVC treatment experiment, the long-term VVC treatment enhances the exercise endurance in weight-loaded swimming mice. The ensemble of results suggests that VVC may offer a promising prospect for enhancing the exercise endurance and alleviating fatigue in humans.

\section{Keywords}

Fatigue, Exercise Endurance, Rhodiola rosea, Eleutherococcus senticosus, Panax quinquefolium

\section{Introduction}

Fatigue, which is a self-limiting response arising from physical and/or mental weariness, can be classified into three categories, namely secondary fatigue, physiologic fatigue and chronic fatigue [1]. Secondary fatigue refers to the weariness secondary to medical condition. While physiologic fatigue refers to an unbalance status of work and rest that can be recovered by taking rest, chronic fatigue is a pathological condition characterized by a persistent (or relapsing) debilitating and clinically unexplained fatigue that leads to a substantial impairment in functional status [2]. Chronic fatigue is a heterogeneous syndrome, characterized by a variety of pathophysiological features including neuroendocrine abnormalities, increased susceptibility to infections, obesity and chronic stress [2]. Despite the diversity of these pathophysiological anomalies, the disruption of structural and functional integrity of mitochondria has been shown to be crucially involved in the development of chronic fatigue, presumably due to an inadequate/inefficient energy supply to skeletal muscle [3] [4]. In order to sustain the energy demands in cytosol of muscle cells, the ATP synthesized in the mitochondria is exchanged with cytosolic ADP by adenine nucleotide translocase (ANT). In addition, the ANT-mediated ATP/ADP exchange was found to be critical for the maintenance of ATP synthase activity [5]. In view of the consequent personal and economic morbidity arising from the negative impacts of fatigue on work performance and social relationships [1], anti-fatigue intervention is urgently sought.

In an effort to develop safe interventions for fatigue, traditional Chinese medicine, which has a long history of use in safeguarding health, has attracted a lot of interest. In the realm of tradition Chinese medicine theories, Qi is a manifestation of energy status of the body [6]. In this connection, "Qi-invigorating" Chinese tonic herbs may produce beneficial effects in fatigue individuals. In support of this, recent studies have demonstrated that an extract of "Yang-invigorating" herb or a compound isolated from "Qi-invigorating" herb can enhance the mitochondrial ATP generation capacity in cultured cardiomyocytes as well as in mitochondria isolated from hearts of drug/herbal extract-treated rats [7] [8]. V-vital capsule (VVC) is an herbal formula comprising three "Qi-invigorating" herbs, namely, the root of Rhodiola rosea, the root of Eleutherococcus senticosus and the root of Panax quinquefolium. With respect to the increased capacity of mitochondrial ATP generation afforded by "Yang/ Qi-invigorating” herbs [7] [8], VVC may offer a prospect for ameliorating physical and/or mental fatigue.

To evaluate the effectiveness of anti-fatigue intervention, force swimming test is a commonly adopted model for assessing the exercise endurance of mice [9] [10]. During the exercise, the supply of metabolic fuel molecules [such as glucose and non-esterified fatty acids (NEFA)] to skeletal muscle [11], the mobilization of energy reserves (such as glycogen in skeletal muscle and liver) [11] as well as the efficiency of mitochondrial respiration in skeletal muscle [12] determine the exercise endurance capacity. In the present study, we endeavored to examine the effect of acute and long treatment with VVC on exercise endurance and the associated changes in various biochemical parameters in mice using a weight-loaded forced swimming test. To assess the availability of fuel molecules, plasma levels of glucose and NEFA were measured. To examine the energy reserve, glycogen levels in skeletal muscle and liver tissues were also measured. In addition, various metabolites such as lactate (arising from the anaerobic glycolysis) and reactive oxygen metabolites (ROM, which is indicative of systemic oxidative stress) in plasma were measured. To assess the energy metabolic status of skeletal muscle, mitochondrial adenine nucleotide translocase (ANT) activity as well as state 3 and state 4 respiration rates of isolated mitochondria of skeletal muscle were assayed. The mitochondrial coupling efficiency was then estimated by computing the ratio of state 3 to state 4 mitochondrial respiratory rates. 


\section{Materials and Methods}

\subsection{Chemicals and Reagents}

Lactate oxidase, horseradish peroxidase, bovine serum albumin (BSA) and 2,2'-azino-bis(3-ethylbenzothiazoline-6-sulfonic acid) diammonium salt (ABST) were purchased from Sigma Chemical Co. (St. Louis, MO, USA). NEFA assay kits were purchased from Wako Pure Chemical Industries, Ltd. (Okasa, Japan). Glucose assay reagent was obtained from Sigma Chemical Co. The VVC were manufactured and supplied by Infinitus (China) Company Ltd. (Guangzhou, China). All other chemicals were of analytical grade.

\subsection{Animal Care}

Male ICR mice (8 - 10 weeks old, 30 - 25 g) were maintained under a 12-hour dark/light cycle at about $22^{\circ} \mathrm{C}$, and allowed food and water ad libitum in the Animal and Plant Care Facility at the Hong Kong University of Science and Technology (HKUST). All experimental protocols were approved by the University Committee on Research Practice at HKUST.

\subsection{Animal Treatment}

In the acute VVC treatment, male ICR mice were randomly assigned to 4 groups, with 10 - 15 mice in each group: (1) control; (2) VVC (0.75 g/kg); (3) VVC (2.5 g/kg); (4) VVC (3.75 g/kg). Control mice received water (vehicle) only. Thirty min post-dosing, the mice were subjected to weight-loaded swimming test. Mice were sacrificed under phenobarbital anesthesia after the swimming test.

In the long-term VVC treatment, male ICR mice were randomly assigned to 6 groups, with $10-15$ mice in each group: (1) non-swimming control; (2) non-swimming VVC (0.075 g/kg); (3) non-swimming VVC (0.25 $\mathrm{g} / \mathrm{kg}$ ); (4) swimming control; (5) swimming VVC (0.075 g/kg); (6) swimming VVC (0.25 g/kg). Immediately after the $1^{\text {st }}$ weight-loaded swimming test (i.e. week 0 ), mice were intragastrically administered with VVC ( 0.075 and $0.25 \mathrm{~g} / \mathrm{kg} /$ day) 5 days per week for 2 weeks (i.e. 10 doses) while control mice received water (vehicle) only. Mice were then subjected to weight-loaded swimming test once a week for 2 weeks (i.e. at week 1 and week 2), at 30 min post-dosing with VVC. Mice were sacrificed under phenobarbital anesthesia after the last swimming test.

\subsection{Weight-Loaded Swimming Test}

The exercise endurance capacity was assessed by weight-loaded swimming test. Swimming exercise was conducted with mice carrying a load of $6 \%$ of their body weight. The exhaustion time was defined as the inability of weight-loaded mice to rise to the water surface for 7 seconds. Swimming exercise was carried out in a tank (20 $\times$ $25 \times 33 \mathrm{~cm}$ ), which was filled with water to a depth of $\sim 28 \mathrm{~cm}$ and maintained at $25^{\circ} \mathrm{C} \pm 1^{\circ} \mathrm{C}$. The tank was shaken at a speed of $40 \mathrm{rpm}$. To avoid the influence of circadian rhythm on physical activity, swimming exercise was done during 11:00 to 17:00, a period in which a minimal variation of endurance capacity has been reported in mice. Hairs of mice were wet with detergent water $(1 \% \mathrm{w} / \mathrm{v})$ to reduce surface tension. The swimming time (second) until exhaustion was recorded.

\subsection{Preparation of Blood/Tissue Samples}

Blood samples were drawn from phenobarbital-anesthetized mice by cardiac excision using syringes rinsed with $0.5 \%$ heparin in saline $(\mathrm{w} / \mathrm{v})$. Plasma samples were obtained by centrifuging whole blood samples at $1500 \times g$ for 10 min at $4^{\circ} \mathrm{C}$. Plasma samples were then subjected to biochemical analysis.

Minced gastrocnemius muscle tissues were digested by collagenase solution $[0.075 \%(\mathrm{w} / \mathrm{v})$ in buffer $]$ at $4^{\circ} \mathrm{C}$ for $20 \mathrm{~min}$. After removing the collagenase solution by centrifugation, the digested muscle tissues were mixed with $20 \mathrm{~mL}$ of ice-cold homogenizing buffer (100 mM KCl, $50 \mathrm{mM}$ MOPS, $10 \mathrm{mM}$ EGTA, pH 7.2) and subjected to homogenization with a Teflon-glass homogenizer at 4,000 rpm for 25 - 30 complete strokes. Then the homogenates were centrifuged at $600 \times g$ for $10 \mathrm{~min}$ at $4^{\circ} \mathrm{C}$. The resultant supernatant was nucleus-free fraction [13].

Mitochondrial pellets were prepared from nucleus-free fractions of muscle homogenates by centrifugation at $9200 \times g$ at $4^{\circ} \mathrm{C}$ for $30 \mathrm{~min}$. The mitochondrial pellets were then resuspended in a buffer containing $250 \mathrm{mM}$ sucrose, $50 \mathrm{mM}$ Tris, pH 7.5 [13]. 


\subsection{Biochemical Analysis}

Plasma fuel molecules. Plasma glucose levels were measured using an assay kit utilizing the coupled hexokinase-catalyzed and glucose-6-phosphate dehydrogenase-catalyzed reactions, with a resultant NAD reduction. Absorbance changes at $340 \mathrm{~nm}$ of the assay mixture were monitored spectrophotometrically by Victor $^{3}$ Mul- $^{-}$ ti-label Counter (Perkin Elmer, Turku, Finland). Plasma NEFA level was measured using assay kit.

Glycogen levels in the liver and skeletal muscle. Liver and skeletal muscle tissues were weighed and then subjected to acidic hydrolysis with $2 \mathrm{M} \mathrm{HCl}$ at $100^{\circ} \mathrm{C}$ for 2 hours. The acidic hydrolyzed tissue samples were neutralized with $2 \mathrm{M} \mathrm{NaOH}$ and then centrifuged at $2150 \times g$ at $4^{\circ} \mathrm{C}$ for $10 \mathrm{~min}$. The glucose level of the supernatant, which contained glucose releasing from glycogen hydrolysis, was measured using assay kit.

Plasma lactate level. Plasma lactate level was measured using a reaction cocktail made up of lactate oxidase, horseradish peroxidase and ABST in a buffer [0.1 M citric acid, $1 \mathrm{mg} / \mathrm{mL}$ BSA, $0.1 \% \mathrm{CaCl}_{2}(\mathrm{w} / \mathrm{v}), 0.02 \%$ sodium azide (w/v), $\mathrm{pH}$ 6.0]. Absorbances at $405 \mathrm{~nm}$ of the assay mixtures were measured spectrophotometrically by Victor ${ }^{3}$ Multi-label Counter.

Plasma reactive oxygen metabolites (ROM) level. The extent of exercise-induced changes in systemic oxidative stress was assessed by the measurement of plasma ROM level. Aliquots $(40 \mu \mathrm{L})$ of plasma samples were mixed with $20 \mu \mathrm{L}$ of $100 \mathrm{mM} \mathrm{N}$, N-dimethyl-p-phenylenediamine (DMPD) and $1.97 \mathrm{~mL}$ of incubation buffer ( $0.1 \mathrm{M}$ sodium acetate, $\mathrm{pH} 4.8$ ). The reaction mixtures were incubated at $37^{\circ} \mathrm{C}$ for $60 \mathrm{~min}$ in dark. Absorbances at $505 \mathrm{~nm}$ of the reaction mixtures were measured using Victor ${ }^{3}$ Multi-Label Counter. The standard calibration curve was obtained by mixing $5 \mu \mathrm{L}$ of tert-butylhydroperoxide (t-BHP) (up to $100 \mu \mathrm{M}$ ) with $20 \mu \mathrm{L}$ phosphate-buffered saline, $5 \mu \mathrm{L}$ of $2.52 \mathrm{mM} \mathrm{FeCl}_{2}$ and $2 \mathrm{~mL}$ of DMPD. The amounts of hydroperoxyl compounds in plasma were estimated from the standard calibration curve and expressed in t-BHP equivalents [14].

Measurement of mitochondrial respiration. Mitochondrial respiratory rate was measured polarographically by a Clark-type oxygen electrode (Hansatech Instruments Ltd., Norfolk, UK) at $30^{\circ} \mathrm{C}$. Mitochondrial fraction $(\sim 0.5$ $\mathrm{mg}$ protein/mL) was incubated in a buffer containing $30 \mathrm{mM} \mathrm{KCl}, 6 \mathrm{mM} \mathrm{MgCl}_{2}, 75 \mathrm{mM}$ sucrose, $1 \mathrm{mM}$ EDTA, $20 \mathrm{mM} \mathrm{KH}_{2} \mathrm{PO}_{4}$ and $0.1 \%$ (w/v) fatty acid-free BSA, $\mathrm{pH}$ 7.0. Substrate solution containing $10 \mathrm{mM}$ glutamate and $2.5 \mathrm{mM}$ malate was added, and after a stable state 2 respiration had been established, state 3 respiration (coupling) was initiated by the addition of ADP (final concentration $0.6 \mathrm{mM}$ ). When all of the added ADP was used up for ATP generation, oligomycin (ATP synthase inhibitor) was added to induce the state 4 respiration (uncoupling). The mitochondrial coupling efficiency was estimated by calculating the ratio of state 3 to state 4 respirations [13].

Adenine nucleotide translocase (ANT) function. ANT function was assessed by noting the transportation of ATP (out) and ADP (in) through mitochondrial inner membrane [4]. The mitochondrial ATP level (a) was first measured. Secondly, the same mitochondrial fraction was mixed with a buffer $(\mathrm{pH}=5.5 \pm 0.2$; serving as an artificial cytosol) containing ADP, which activates the ANT to transport ADP to the mitochondrial matrix. After 10 min of incubation, the mitochondrial ATP level (b) was measured again. The value of $\mathrm{ANT}_{\text {in }}$ was estimated by measuring the fractional increase in mitochondrial ATP as follow: $\mathrm{ANT}_{\text {in }}=[(\mathrm{b}-\mathrm{a}) / \mathrm{a}]$. Thirdly, the same mitochondrial fraction was mixed with a buffer $(\mathrm{pH}=8.9 \pm 0.2)$ containing no ADP, which activates the ANT to transport ATP from the mitochondrial matrix to the artificial cytosol. After 10 min of incubation, the mitochondrial ATP level was measured again (c). The value of $\mathrm{ANT}_{\text {out }}$ was estimated by measuring the fractional decrease in ATP as follows: $\mathrm{ANT}_{\text {out }}=[(\mathrm{c}-\mathrm{a}) / \mathrm{a}]$

\subsection{Statistical Analysis}

Data were analyzed by one-way Analysis of Variance (ANOVA), except the data of the swimming time in long-term VVC treatment, were analyzed by mixed ANOVA. Post-hoc multiple comparisons were performed using Least Significant Difference. P values $<0.05$ were regarded as statistically significant.

\section{Results}

\subsection{Effects of Acute VVC Treatment on Weight-Loaded Swimming Time in Mice}

Acute VVC treatment (2.5 and $3.75 \mathrm{~g} / \mathrm{kg}$ ) 30 min prior to weight-loaded swimming increased the weight-loaded swimming time by $\sim 1$-fold (104\% and 104\%, respectively) until exhaustion in mice (Figure 1). 


\subsection{Effects of Acute VVC Treatment on Plasma Levels of Fuel Molecules in Non-Swimming and Weight-Loaded Swimming Mice}

Acute VVC treatment $(0.75,2.5$ and $3.75 \mathrm{~g} / \mathrm{kg})$ dose-dependently increased plasma glucose level $(22 \%$, 40\% and 54\%, respectively) in non-swimming mice (Figure 2). However, acute VVC treatment decreased the plasma NEFA level (29\%, 33\% and 47\%, respectively) in a dose-dependent manner. Weight-loaded swimming until exhaustion decreased plasma levels of glucose (25\%) and NEFA (32\%) when compared with the non-swimming control. Acute VVC treatment increased plasma glucose level (44\%, $46 \%$ and 31\%, respectively) and decreased plasma NEFA level (21\% and 25\% at 2.5 and $3.75 \mathrm{~g} / \mathrm{kg}$, respectively) in weight-loaded swimming mice.

\subsection{Effects of Acute VVC Treatment on Skeletal/Hepatic Glycogen Levels in Non-Swimming and Weight-Loaded Swimming Mice}

Acute VVC treatment did not produce any detectable changes in hepatic/skeletal muscle glycogen in

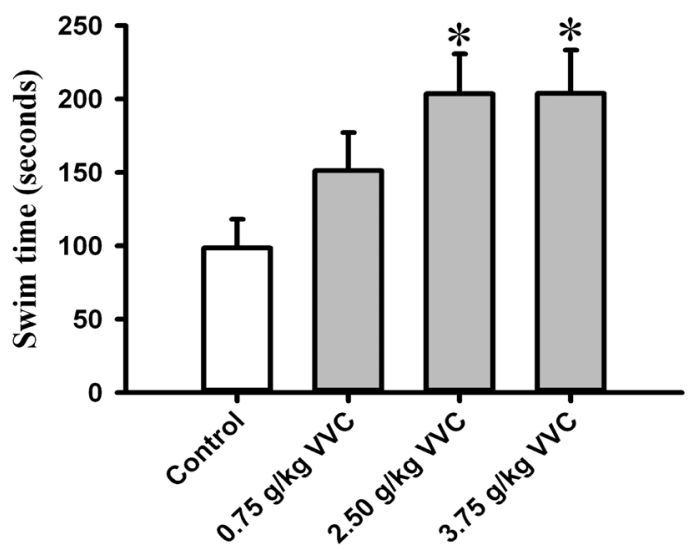

Figure 1. Effects of acute VVC treatment on weight-loaded swimming time in mice. Mice were intragastrically administered with VVC $(0.75,2.5$ and $3.75 \mathrm{~g} / \mathrm{kg})$. At 30 minutes post-dosing, the mice were subjected to weight-loaded swimming test, as described in Materials and Methods. The swimming times of mice until exhaustion were recorded. Data were expressed as swimming time (second). Value given are means \pm SEM, with n = 10 - 15. * Significantly different from drug-untreated control.
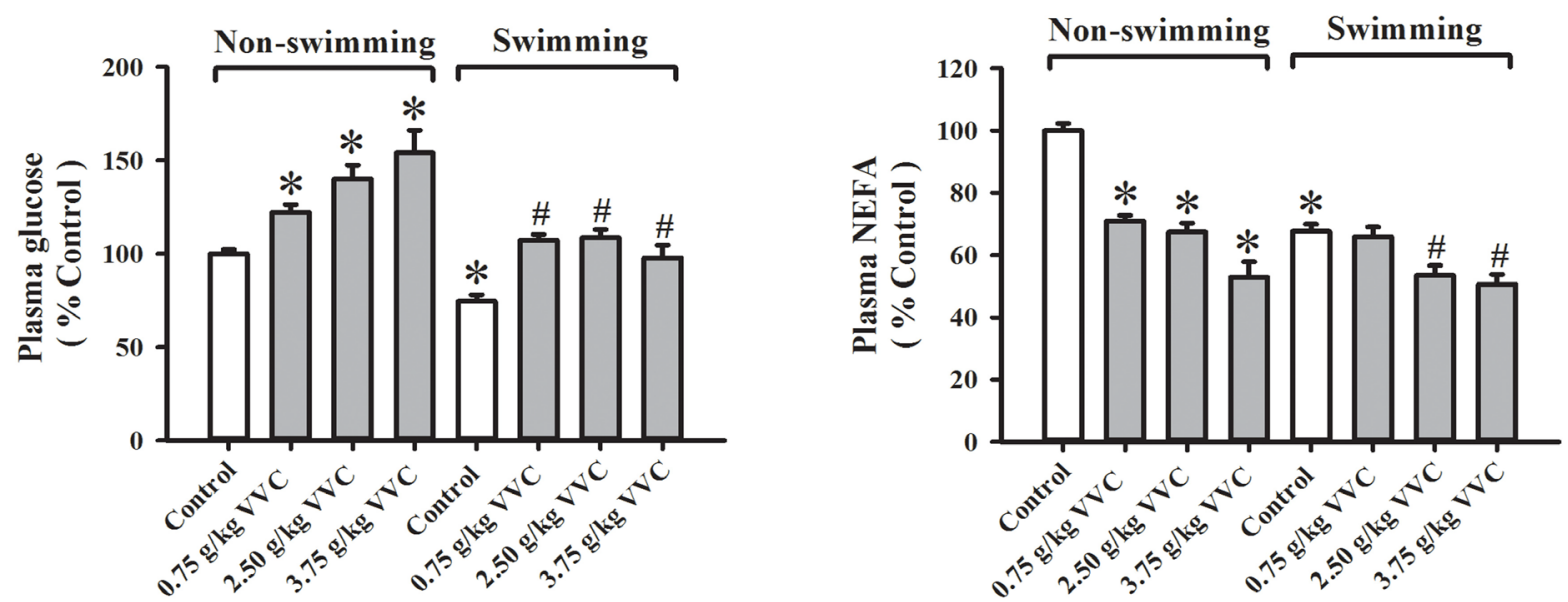

Figure 2. Effects of acute VVC treatment on plasma levels of fuel molecules in non-swimming and weight-loaded swimming mice. Mice were sacrificed after weight-loaded swimming until exhaustion. Plasma glucose level (Non-swimming control $=71.62 \pm 2.61 \mathrm{mg} / \mathrm{mL})$ and plasma non-esterified fatty acid $(\mathrm{NEFA})$ level (Non-swimming control = $1.44 \pm 0.05 \mathrm{mEq} / \mathrm{L}$ ) were measured, as described in Materials and Methods. Data were expressed as \% control, by normalizing with the value of non-swimming control. Value given are means \pm SEM, with $n=10-15$ * Significantly different from non-swimming control; \# Significantly different from swimming control. 
non-swimming mice. Weight-loaded swimming until exhaustion depleted skeletal muscle (49\%) but not hepatic glycogen level in mice (Figure 3). Acute VVC treatment caused increases in skeletal muscle and hepatic glycogen levels in weight-loaded swimming mice, with maximal stimulation being $60 \%$ and $87 \%$ at the doses of 2.5 and $3.75 \mathrm{~g} / \mathrm{kg}$, respectively.

\subsection{Effects of Acute VVC Treatment on Plasma Levels of Metabolites in Non-Swimming and Weight-Loaded Swimming Mice}

Acute VVC treatment did not change plasma lactate levels in non-swimming mice. Weight-loaded swimming until exhaustion increased plasma lactate level (45\%) (Figure 4), which was associated with the decrease in
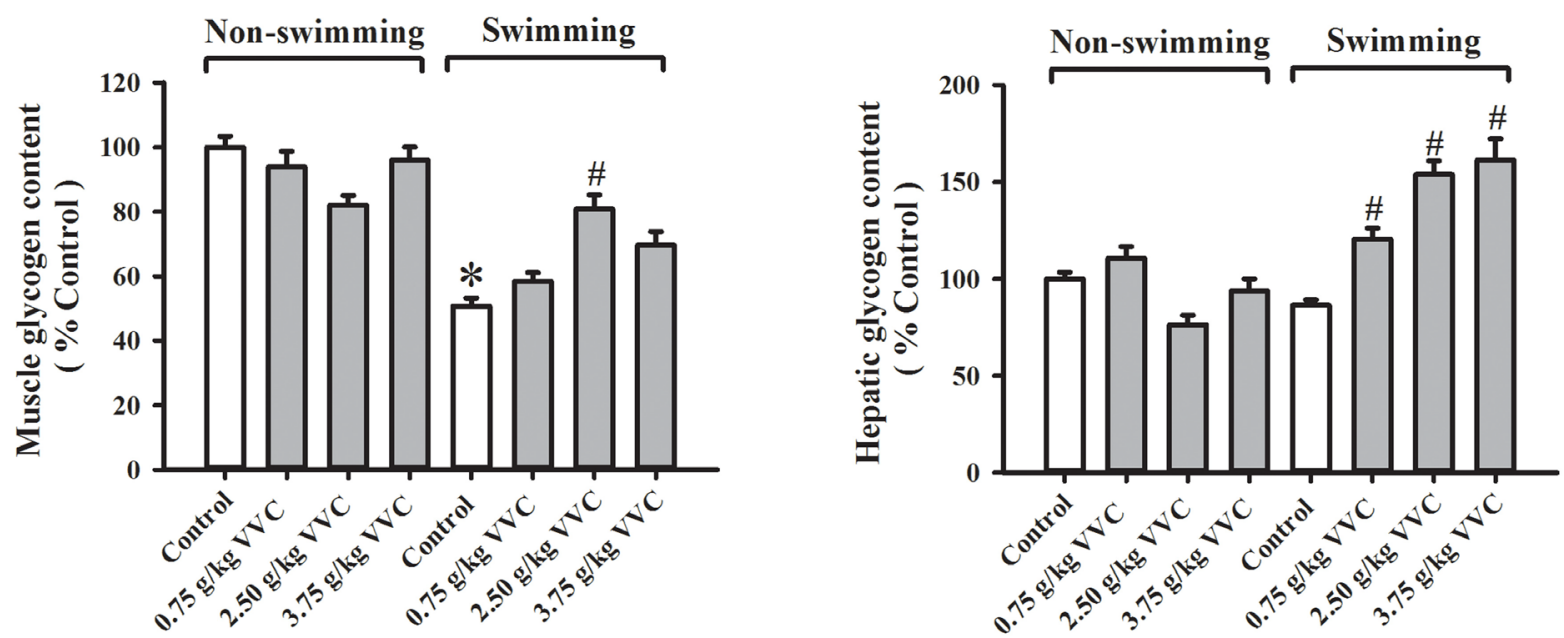

Figure 3. Effects of acute VVC treatment on skeletal/hepatic glycogen levels in non-swimming and weight-loaded swimming mice. Mice were sacrificed after weight-loaded swimming until exhaustion. Skeletal glycogen level (Non-swimming control $=0.52 \pm 0.03 \mu \mathrm{mol}$ glucosyl unit/unit $\mathrm{g}$ tissue) and hepatic glycogen level (Non-swimming control $=5.95 \pm 0.41$ $\mu \mathrm{mol}$ glucosyl unit/unit g tissue) were measured, as described in Materials and Methods. Data were expressed as \% control, by normalizing with the value of non-swimming control. Value given are means \pm SEM, with $n=10-15$. * Significantly different from non-swimming control; \# Significantly different from swimming control.
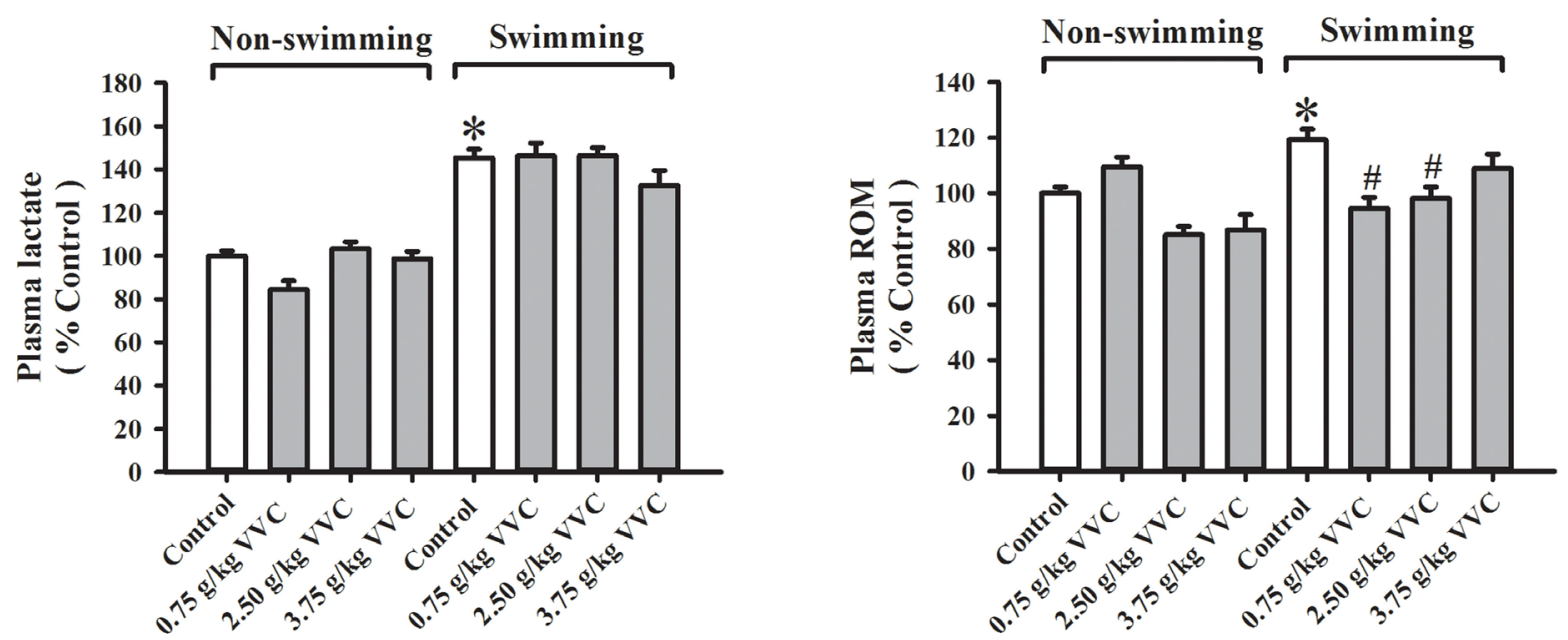

Figure 4. Effects of acute VVC treatment on plasma levels of metabolites in non-swimming and weight-loaded swimming mice. Mice were sacrificed after weight-loaded swimming until exhaustion. Plasma lactate level (Non-swimming control = $4.33 \pm 0.24 \mathrm{mM}$ ) and plasma reactive oxygen metabolites (ROM) level (Non-swimming control $=105 \pm 4.39 \mu \mathrm{mol} \mathrm{tBHP}$ equivalent) were measured, as described in Materials and Methods. Data were expressed as \% control, by normalizing with the value of non-swimming control. Value given are means \pm SEM, with $n=10-15$. Significantly different from nonswimming control; \# Significantly different from swimming control. 
plasma glucose level in weight-loaded swimming. Despite the fact that acute VVC treatment increased in plasma glucose level in weight-loaded swimming mice, plasma lactate levels were not affected. Acute VVC treatment also did not alter plasma ROM level in non-swimming mice. Weight-loaded swimming until exhaustion increased plasma ROM level (19\%) in mice which was found to be reduced by acute VVC treatment (21\% and $18 \%$ at 0.75 and $2.5 \mathrm{~g} / \mathrm{kg}$, respectively).

\subsection{Effects of Acute VVC Treatment on Mitochondrial Functional Status of Skeletal Muscle in Non-Swimming and Weight-Loaded Swimming Mice}

Acute VVC treatment did not produce any detectable change in the mitochondrial coupling efficiency of skeletal muscle in non-swimming mice (Figure 5). Weight-loaded swimming until exhaustion decreased mitochondrial coupling efficiency (24\%) in mouse skeletal muscle. Acute VVC treatment at $0.75 \mathrm{~g} / \mathrm{kg}$ significantly increased mitochondrial coupling efficiency (37\%) in weight-loaded swimming mice. Acute VVC treatment $(0.75,2.5$ and
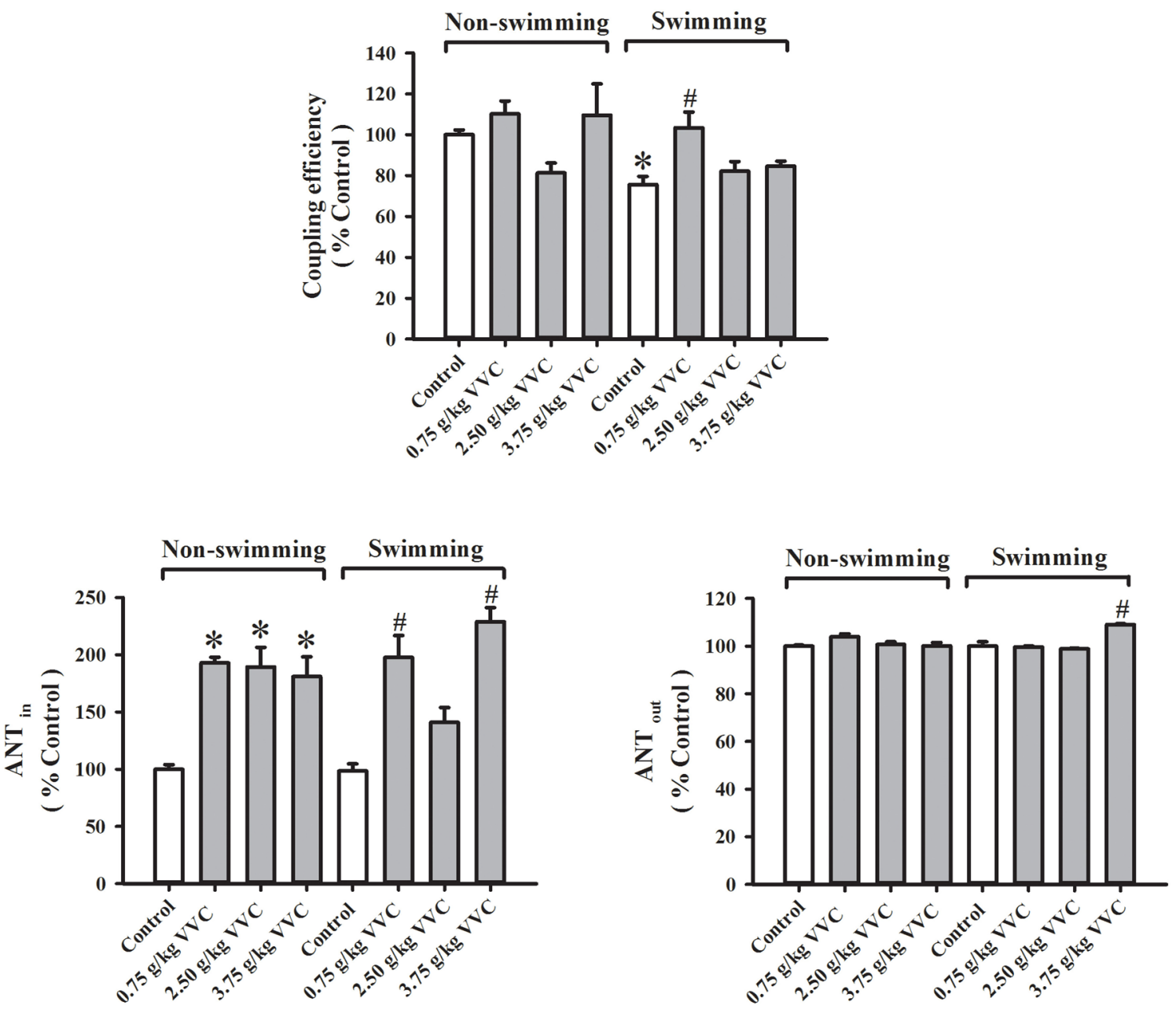

Figure 5. Effects of acute VVC treatment on mitochondrial functional status of skeletal muscle in non-swimming and weight-loaded swimming mice. Mice were sacrificed after weight-loaded swimming until exhaustion. Mitochondrial respiratory rates of skeletal muscle was measured polarographically by a Clark-type oxygen electrode at $30^{\circ} \mathrm{C}$. The coupling efficiency was estimated by calculating the ratio of state 3 to state 4 respirations (Non-swimming control = $2.51 \pm 0.07$ ). The effectiveness of adenosine nucleotide translocase (ANT) of skeletal muscle was also assessed, as described in Materials and Methods. $\mathrm{ANT}_{\text {in }}$ (which indicates the effectiveness of ADP influx in mitochondria; Non-swimming control $=9.02 \pm 1.00$ ) and $\mathrm{ANT}_{\text {out }}$ (which indicates the effectiveness of ATP outflux from mitochondria; Non-swimming control $=0.94 \pm 0.01$ ) were measured. Data were expressed as \% control, by normalizing with the value of non-swimming control. Values given are means \pm SEM, with $n=5-10$. *Significantly different from non- swimming control; \# Significantly different from swimming control. 
$3.75 \mathrm{~g} / \mathrm{kg}$ ) significantly increased mitochondrial $\mathrm{ANT}_{\text {in }}$ activity (93\%, 89\% and 81\%, respectively) in skeletal muscle of non-swimming mice, but it did not affect the $\mathrm{ANT}_{\text {out }}$ activity. While weight-loaded swimming until exhaustion did not alter mitochondrial $\mathrm{ANT}_{\text {in/out }}$ activities, acute VVC treatment increased the $\mathrm{ANT}_{\text {in }}$ activity (101\% and $92 \%$ at 0.75 and $3.75 \mathrm{~g} / \mathrm{kg}$, respectively) in weight-loaded swimming mice. The $\mathrm{ANT}_{\text {out }}$ activity seemed to be slightly but significantly increased $(9 \%)$ in acute VVC-treated $(3.75 \mathrm{~g} / \mathrm{kg})$ and weight-loaded swimming mice.

\subsection{Effects of Long-Term VVC Treatment on the Weight-Loaded Swimming Time of Mice}

Long-term treatment $(0.075$ and $0.25 \mathrm{~g} / \mathrm{kg})$ for 2 weeks progressively increased the weight-loaded swimming time of mice, with the effect produced by the low dose of VVC being more prominent at 1 week post-treatment and the extent of prolongation in weight-loaded swimming time being $35.6 \%$ for both doses at 2 weeks posttreatment (Figure 6).

\subsection{Effects of Long-Term VVC Treatment on Various Biochemical Parameters in Non-Swimming and Weight-Loaded Swimming Mice}

Long-term VVC treatment did not produce any significant changes in the levels of plasma glucose, muscle glycogen, plasma metabolites as well as mitochondrial coupling efficiency in skeletal muscle of non-swimming mice, except a decrease in plasma NEFA (15\% at $0.25 \mathrm{~g} / \mathrm{kg}$ ), increases in hepatic glycogen ( $29 \%$ and $25 \%$ at 0.075 and $0.25 \mathrm{~g} / \mathrm{kg}$, respectively) as well as a dose-dependent increase in mitochondrial $\mathrm{ANT}_{\text {in }}$ activity in skeletal muscle ( $95 \%$ and $115 \%$ at 0.075 and $0.25 \mathrm{~g} / \mathrm{kg}$, respectively) (Table 1). Consistent with the results obtained from acute VVC treatment, weight-loaded swimming until exhaustion significantly decreased the plasma glucose as well as NEFA levels (25\% and 32\%, respectively), skeletal muscle glycogen level (46\%) and hepatic glycogen level (37\%). The weight-loaded swimming-induced depletions in plasma fuel molecules and energy reserves were associated with increases in plasma levels of metabolites, such as lactate (30\%) and ROM (39\%). While the long-term VVC treatment at low dose increased the mitochondrial $\mathrm{ANT}_{\text {in }}$ activity (79\% vs. swimming control) in skeletal muscle of weight-loaded swimming mice, the long-term VVC treatment at high dose further increased the glycogen level (44\% vs. swimming control) as well as mitochondrial coupling efficiency ( $83 \%$ vs swimming control) in skeletal muscle. The plasma ROM level was reduced ( $19 \%$ vs. swimming control) in VVC-treated $(0.25 \mathrm{~g} / \mathrm{kg})$ weight-loaded swimming mice.

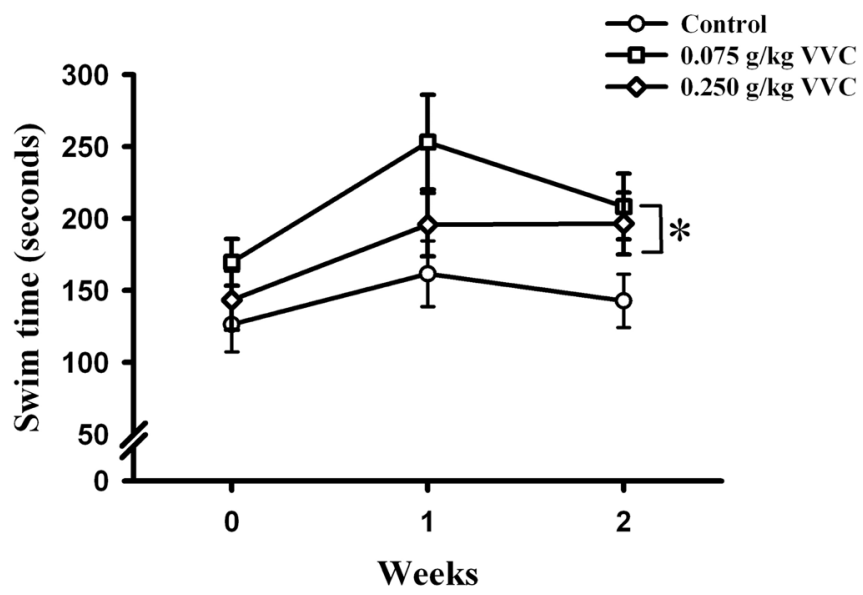

Figure 6. Effects of long-term VVC treatment on the weight-loaded swimming time of mice. Mice were subjected to weight-loaded swimming test every week (including the week 0) during the 2-week period of experiment, as described in Materials and Methods. The swimming times of mice until exhaustion were recorded. Immediately after the $1^{\text {st }}$ weight-loaded swimming test (i.e. week 0 ), mice were intragastrically administered with VVC ( 0.075 and $0.25 \mathrm{~g} / \mathrm{kg} /$ day) 5 days per week for 2 weeks (i.e. 10 doses), while control mice received water (vehicle) only. Data were expressed as swim time (second). Value given are means \pm SEM, with $n=10-15$. * Significantly different from drug-untreated control. 
Table 1. Effects of long-term VVC treatment on various biochemical parameters in non-swimming and weight-loaded swimming mice.

\begin{tabular}{|c|c|c|c|c|c|c|}
\hline & \multicolumn{3}{|c|}{ Non-Swim } & \multicolumn{3}{|c|}{ Swim } \\
\hline & \multirow[t]{2}{*}{ Control } & \multicolumn{2}{|c|}{ VVC } & \multirow[t]{2}{*}{ Control } & \multicolumn{2}{|c|}{ VVC } \\
\hline & & $0.075 \mathrm{~g} / \mathrm{kg}$ & $0.25 \mathrm{~g} / \mathrm{kg}$ & & $0.075 \mathrm{~g} / \mathrm{kg}$ & $0.25 \mathrm{~g} / \mathrm{kg}$ \\
\hline \multicolumn{7}{|l|}{ Fuel molecules } \\
\hline Plasma glucose & $100 \pm 2.4$ & $108 \pm 4.9$ & $107 \pm 3.2$ & $74.5 \pm 3.3^{\mathrm{a}}$ & $61.0 \pm 2.1$ & $68.0 \pm 3.5$ \\
\hline Plasma NEFAs & $100 \pm 2.4$ & $98.1 \pm 3.0$ & $84.8 \pm 2.2^{\mathrm{a}}$ & $68.2 \pm 3.3^{\mathrm{a}}$ & $65.8 \pm 209$ & $74.3 \pm 3.2$ \\
\hline \multicolumn{7}{|l|}{ Energy reserves } \\
\hline Muscle glycogen & $100 \pm 2.2$ & $93.3 \pm 2.5$ & $99.5 \pm 2.7$ & $53.8 \pm 2.1^{\mathrm{a}}$ & $65.5 \pm 4.1$ & $77.6 \pm 4.4^{\mathrm{b}}$ \\
\hline Hepatic glycogen & $100 \pm 2.3$ & $129 \pm 5.7^{\mathrm{a}}$ & $125 \pm 3.9^{\mathrm{a}}$ & $62.6 \pm 2.4^{\mathrm{a}}$ & $74.7 \pm 3.8$ & $84.1 \pm 4.5$ \\
\hline \multicolumn{7}{|l|}{ Metabolites } \\
\hline Plasma lactate & $100 \pm 1.7$ & $91.0 \pm 4.0$ & $90.9 \pm 3.7$ & $130 \pm 4.1^{\mathrm{a}}$ & $123 \pm 3.4$ & $115 \pm 2.8$ \\
\hline ROM & $100 \pm 2.8$ & $95.9 \pm 2.9$ & $90.1 \pm 3.3$ & $139 \pm 5.2^{\mathrm{a}}$ & $126 \pm 2.9$ & $113 \pm 2.9^{b}$ \\
\hline \multicolumn{7}{|c|}{$\begin{array}{l}\text { Mitochondrial functional status } \\
\text { in skeletal muscle }\end{array}$} \\
\hline Coupling efficiency & $100 \pm 2.7$ & $88.1 \pm 14.7$ & $87.7 \pm 5.4$ & $67.1 \pm 10.2$ & $90.9 \pm 5.1$ & $123 \pm 17.8^{\mathrm{b}}$ \\
\hline $\mathrm{ANT}_{\text {in }}$ & $100 \pm 4.5$ & $195 \pm 15.6^{\mathrm{a}}$ & $215 \pm 22.0^{\mathrm{a}}$ & $144 \pm 14.2$ & $258 \pm 32.8^{\mathrm{b}}$ & $110 \pm 7.7$ \\
\hline $\mathrm{ANT}_{\text {out }}$ & $100 \pm 0.4$ & $101 \pm 0.2$ & $102 \pm 0.8$ & $99.8 \pm 0.2$ & $100 \pm 0.1$ & $101 \pm 0.3$ \\
\hline
\end{tabular}

a. significantly different from non-swimming control; b. significantly different from swimming control.

\section{Discussion}

Acute VVC treatment increased the exercise endurance capacity in mice, as indicated by the increase in the swimming time of weight-loaded swimming mice. The ability of VVC to enhance exercise endurance is consistent with the experimental observations that all component herbs of VVC (i.e., the root of Rhodiola rosea, Eleutherococcus senticosus and Panax quinquefolium) independently increased the exercise endurance capacity in rodents. In this regard, the treatment with Rhodiola rosea extract was found to increase the swimming performance in both weight-unloaded and loaded rats [15] [16]. While the Eleutherococcus senticosus extract could increase the swimming time in forced swimming mice [17], the treatment with a protein fraction (containing proteins ranging from 8 - $66 \mathrm{kDa}$ ) isolated from Panax quinquefolium was able to increase the exercise endurance in weight-loaded swimming mice [18].

Force swimming-induced exhaustion, as observed in the present and other studies [9] [10], was found to be accompanied by depletions in plasma fuel molecules, suggestive of their involvement in muscle fatigue. In the present study, acute VVC treatment was found to increase the plasma level of glucose, with a concomitant decrease in plasma NEFA level, in both non-swimming and swimming mice. While the adipose tissue-derived NEFA is the major oxidative fuel molecule in skeletal muscle during low intensity exercise (such as weightunloaded swimming) [19], plasma glucose is the major fuel molecule in skeletal muscle during high intensity exercise (such as weight-loaded swimming) [20]. As such, the enhancement in exercise endurance, as assessed by weight-loaded swimming, afforded by VVC may be due to the increase in plasma glucose supply to skeletal muscle for the anaerobic glycolysis. The lactate arising from anaerobic glycolysis then can undergo Cori cycle in the liver, with a resultant synthesis of glucose or glycogen. Given that high intensity exercise was found to time-dependently increase the plasma lactate level in rodents [21], VVC-treated mice, of which the swimming time is longer than those of VVC-untreated mice, should be expected to exhibit a higher plasma lactate level. However, VVC treatment did not increase the plasma lactate level in swimming mice, suggesting that plasma lactate may be utilized for gluconeogenesis and/or glycogen synthesis in the liver. This postulation is further strengthened by the observation that the glycogen levels in skeletal muscle and liver were elevated in VVC- 
treated weight-loaded swimming mice.

The increased production of metabolites [e.g. $\mathrm{H}^{+}$ion, lactate and reactive oxygen species (ROS)] during high intensity exercise was also found to be associated with muscle fatigue [22]. High plasma levels of $\mathrm{H}^{+} /$lactate were conventionally considered as the major cause of muscle fatigue [23]. As mentioned earlier, VVC may facilitate the metabolism of lactate in the liver, leading to a postponed increase in plasma lactate level, which in turn enhances the exercise endurance. Recent studies have shown that low pH [24] [25] and high lactate level [26] [27] may have less inhibitory effect on the muscle contraction than that of previously assumed. The muscle fatigue may be mainly caused by the reduction of sarcoplasmic reticulum (SR) $\mathrm{Ca}^{2+}$ release [28]. With this notion in mind, ROS, which could reduce the maximum $\mathrm{Ca}^{2+}$-activated force in skeletal muscle, the $\mathrm{SR} \mathrm{Ca}^{2+}$ release and the $\mathrm{Ca}^{2+}$ sensitivity of contractile proteins [28], are hypothesized to play a critical role in muscle fatigue. In this connection, the acute VVC treatment-induced amelioration of systemic oxidative stress in swimming mice, as evidenced by a reduction of plasma ROM, may also contribute to the enhancement on exercise endurance. In support of this, a constituent of VVC, Rhodiola rosea, was also found to reduce swimming-enhanced oxidative stress in rat, possibly via its ROS scavenging capability and induction of the antioxidant defense system [15].

Acute VVC treatment improved the mitochondrial function in swimming mice, as indicated by increases in mitochondrial coupling efficiency and $\mathrm{ANT}_{\text {in/out }}$ activities. An earlier study suggested that the mitochondrial function in skeletal muscle was positively correlated with the physical exercise performance in humans [29]. In this regard, the VVC-induced enhancement in exercise endurance may also be attributed to the improvement of mitochondrial function in skeletal muscle of swimming mice. In addition, a recent study showed that the PGC- $1 \alpha$-mediated activation of ANT reduced the production of mitochondrial ROS in cultured endothelial cells, with a resultant protection against oxidant-induced apoptosis [30]. Therefore, the activation of ANT by VVC may also be involved in the attenuation of systemic oxidative stress.

Consistent with the results obtained in acute VVC treatment, we further demonstrated that a long-term and low dose VVC treatment enhanced the exercise endurance in weight-loaded swimming mice, with an associated increase in $\mathrm{ANT}_{\text {in }}$ activity at a low dose of treatment. However, varied effects on plasma fuel molecules, plasma metabolites, glycogen levels in skeletal muscle and liver, as well as mitochondrial functional status were observed between the acute and long-term treatment, which may possibly be related to the differences in the dose and duration of VVC treatment.

\section{Conclusion}

In conclusion, both acute and long-term VVC treatments were found to enhance the exercise endurance in weight-loaded swimming mice. The enhancement in exercise endurance afforded by acute VVC treatment was associated with the increase in plasma fuel molecule, the amelioration of systemic oxidative stress, and the improvement in mitochondrial function of skeletal muscle. The ensemble of results suggests that VVC may offer a promising prospect for enhancing the exercise endurance and alleviating fatigue in humans.

\section{References}

[1] Rosenthal, T.C., Majeroni, B.A., Pretorius, R. and Malik, K. (2008) Fatigue: An Overview. American Family Physician, 78, 1173-1179.

[2] Afari, N. and Buchwald, D. (2003) Chronic Fatigue Syndrome: A Review. The American Journal of Psychiatry, 160, 221-236. http://dx.doi.org/10.1176/appi.ajp.160.2.221

[3] Plioplys, A.V. and Plioplys, S. (1995) Electron-Microscopic Investigation of Muscle Mitochondria in Chronic Fatigue Syndrome. Neuropsychobiology, 32, 175-181. http://dx.doi.org/10.1159/000119233

[4] Myhill, S., Booth, N.E. and McLaren-Howard, J. (2009) Chronic Fatigue Syndrome and Mitochondrial Dysfunction. International Journal of Clinical and Experimental Medicine, 2, 1-6.

[5] Vander Heiden, M.G., Chandel, N.S., Schumacker, P.T. and Thompson, C.B. (1999) Bcl-xL Prevents Cell Death Following Growth Factor Withdrawal by Facilitating Mitochondrial ATP/ADP Exchange. Molecular Cell, 3, 159-167. http://dx.doi.org/10.1016/S1097-2765(00)80307-X

[6] Zhang, D. and Wu, X. (1991) Chapter 5 Qi, Blood, Body Fluid, Essence of Life and Spirit. In: Liu, Y., Ed., The Basic Knowledge of Traditional Chinese Medicine, Hai Feng Publishing Co., Hong Kong, 49-53.

[7] Wong, H.S., Chen, N., Leong, P.K. and Ko, K.M. (2013) $\beta$-Sitosterol Enhances Cellular Glutathione Redox Cycling by Reactive Oxygen Species Generated From Mitochondrial Respiration: Protection Against Oxidant Injury in H9c2 Cells 
and Rat Hearts. Phytotherapy Research, 28, 999-1006. http://dx.doi.org/10.1002/ptr.5087

[8] Chiu, P.Y. and Ko, K.M. (2003) Time-Dependent Enhancement in Mitochondrial Glutathione Status and ATP Generation Capacity by Schisandrin B Treatment Decreases the Susceptibility of Rat Hearts to Ischemia-Reperfusion Injury. Biofactors, 19, 43-51. http://dx.doi.org/10.1002/biof.5520190106

[9] Su, K.Y., Yu, C.Y., Chen, Y.W., Huang, Y.T., Chen, C.T., Wu, H.F. and Chen, Y.L. (2014) Rutin, a Flavonoid and Principal Component of Saussurea Involucrata, Attenuates Physical Fatigue in a Forced Swimming Mouse Model. International Journal of Medical Sciences, 11, 528-537. http://dx.doi.org/10.7150/ijms.8220

[10] Chen, J.C., Hsiang, C.Y., Lin, Y.C. and Ho, T.Y. (2014) Deer Antler Extract Improves Fatigue Effect through Altering the Expression of Genes Related to Muscle Strength in Skeletal Muscle of Mice. Evidence-Based Complementary and Alternative Medicine, 2014, Article ID: 540580. http://dx.doi.org/10.1155/2014/540580

[11] Holloszy, J.O. and Kohrt, W.M. (1996) Regulation of Carbohydrate and Fat Metabolism during and after Exercise. Annual Review of Nutrition, 16, 121-138. http://dx.doi.org/10.1146/annurev.nu.16.070196.001005

[12] Conley, K.E., Jubrias, S.A., Cress, M.E. and Esselman, P.C. (2013) Elevated Energy Coupling and Aerobic Capacity Improves Exercise Performance in Endurance-Trained Elderly Subjects. Experimental Physiology, 98, 899-907. http://dx.doi.org/10.1113/expphysiol.2012.069633

[13] Leong, P.K., Leung, H.Y., Wong, H.S., Chen, J.H., Chan, W.M., Ma, C.W., Yang, Y.T. and Ko, K.M. (2014) LongTerm Treatment with an Herbal Formula MCC Ameliorates Obesity-Associated Metabolic Dysfunction in High Fat Diet-Induced Obese Mice: A Comparative Study among MCC and Various Combinations of Its Constituents. Chinese Medicine, 5, 34-46. http://dx.doi.org/10.4236/cm.2014.51005

[14] Leong, P.K., Chen, N., Chiu, P.Y., Leung, H.Y., Ma, C.W., Tang, Q.T. and Ko, K.M. (2010) Long-Term Treatment with Shengmai San-Derived Herbal Supplement (Wei Kang Su) Enhances Antioxidant Response in Various Tissues of Rats with Protection against Carbon Tetrachloride Hepatotoxicity. Journal of Medicinal Food, 13, 427-438. http://dx.doi.org/10.1089/jmf.2009.1296

[15] Huang, S.C., Lee, F.T., Kuo, T.Y., Yang, J.H. and Chien, C.T. (2009) Attenuation of Long-Term Rhodiola rosea Supplementation on Exhaustive Swimming-Evoked Oxidative Stress in the Rat. Chinese Journal of Physiology, 52, 316324. http://dx.doi.org/10.4077/CJP.2009.AMH029

[16] Lee, F.T., Kuo, T.Y., Liou, S.Y. and Chien, C.T. (2009) Chronic Rhodiola rosea Extract Supplementation Enforces Exhaustive Swimming Tolerance. The American Journal of Chinese Medicine, 37, 557-572. http://dx.doi.org/10.1142/S0192415X09007053

[17] Kimura, Y. and Sumiyoshi, M. (2004) Effects of Various Eleutherococcus senticosus Cortex on Swimming Time, Natural Killer Activity and Corticosterone Level in Forced Swimming Stressed Mice. Journal of Ethnopharmacology, 95, 447-453. http://dx.doi.org/10.1016/j.jep.2004.08.027

[18] Qi, B., Liu, L., Zhang, H., Zhou, G.X., Wang, S., Duan, X.Z., Bai, X.Y., Wang, S.M. and Zhao, D.Q. (2014) Anti-Fatigue Effects of Proteins Isolated from Panax quinquefolium. Journal of Ethnopharmacology, 153, 430-434. http://dx.doi.org/10.1016/j.jep.2014.02.045

[19] Frayn, K.N. (2010) Fat as a Fuel: Emerging Understanding of the Adipose Tissue-Skeletal Muscle Axis. Acta Physiologica, 199, 509-518. http://dx.doi.org/10.1111/j.1748-1716.2010.02128.x

[20] Jeppesen, J. and Kiens, B. (2012) Regulation and Limitations to Fatty Acid Oxidation during Exercise. Journal of Physiology, 590, 1059-1068.

[21] Kato, M., Kurakane, S., Nishina, A., Park, J. and Chang H. (2013) The Blood Lactate Increase in High Intensity Exercise Is Depressed by Acanthopanax sieboldianus. Nutrients, 5, 4134-4144. http://dx.doi.org/10.3390/nu5104134

[22] Green, H.J. (1997) Mechanisms of Muscle Fatigue in Intense Exercise. Journal of Sports Sciences, 15, 247-256. http://dx.doi.org/10.1080/026404197367254

[23] Fitts, R.H. (1994) Cellular Mechanisms of Muscle Fatigue. Physiological Reviews, 74, 49-94.

[24] Cady, E.B., Jones, D.A., Lynn, J. and Newham, D.J. (1989) Changes in Force and Intracellular Metabolites during Fatigue of Human Skeletal Muscle. Journal of Physiology, 418, 311-325.

[25] Stary, C.M. and Hogan, M.C. (2005) Intracellular pH during Sequential, Fatiguing Contractile Periods in Isolated Single Xenopus Skeletal Muscle Fibers. Journal of Applied Physiology, 99, 308-312. http://dx.doi.org/10.1152/japplphysiol.01361.2004

[26] Karlsson, J., Funderburk, C.F., Essen, B. and Lind, A.R. (1975) Constituents of Human Muscle in Isometric Fatigue. Journal of Applied Physiology, 38, 208-211.

[27] Van Beekvelt, M.C., Drost, G., Rongen, G., Stegeman, D.F., Van Engelen, B.G. and Zwarts, M.J. (2006) Na ${ }^{+}-K^{+}-$ ATPase Is Not Involved in the Warming-Up Phenomenon in Generalized Myotonia. Muscle \& Nerve, 33, 514-523. http://dx.doi.org/10.1002/mus.20483 
[28] Allen, D.G., Lamb, G.D. and Westerblad, H. (2008) Skeletal Muscle Fatigue: Cellular Mechanisms. Physiological Reviews, 88, 287-332. http://dx.doi.org/10.1152/physrev.00015.2007

[29] Jacobs, R.A., Flück, D., Bonne, T.C., Bürgi, S., Christensen, P.M., Toigo, M. and Lundby C. (1985) Improvements in Exercise Performance with High-Intensity Interval Training Coincide with an Increase in Skeletal Muscle Mitochondrial Content and Function. Journal of Applied Physiology, 115, 785-793. http://dx.doi.org/10.1152/japplphysiol.00445.2013

[30] Won, J.C., Park, J.Y., Kim, Y.M., Koh, E.H., Seol, S., Jeon, B.H., Han, J., Kim, J.R., Park, T.S., Choi, C.S., Lee, W.J., Kim, M.S., Lee, I.K., Youn, J.H. and Lee, K.U. (2010) Peroxisome Proliferator-Activated Receptor- $\gamma$ Coactivator 1- $\alpha$ Overexpression Prevents Endothelial Apoptosis by Increasing ATP/ADP Translocase Activity. Thrombosis, and Vascular Biology, 30, 290-297. http://dx.doi.org/10.1161/ATVBAHA.109.198721

\section{List of Abbreviations}

Adenine nucleotide translocase (ANT); Analysis of Variance (ANOVA); 2,2'-azino-bis(3-ethylbenzothiazoline-6-sulfonic acid) diammonium salt (ABST); Bovine serum albumin (BSA); tert-Butylhydroperoxide (t-BHP); N, N-dimethyl-p-phenylenediamine (DMPD); Non-esterified fatty acid (NEFA); Reactive oxygen metabolites (ROM); Reactive oxygen species (ROS); Sarcoplasmic reticulum (SR); V-vital capsule (VVC). 
Scientific Research Publishing (SCIRP) is one of the largest Open Access journal publishers. It is currently publishing more than 200 open access, online, peer-reviewed journals covering a wide range of academic disciplines. SCIRP serves the worldwide academic communities and contributes to the progress and application of science with its publication.

Other selected journals from SCIRP are listed as below. Submit your manuscript to us via either submit@scirp.org or Online Submission Portal.
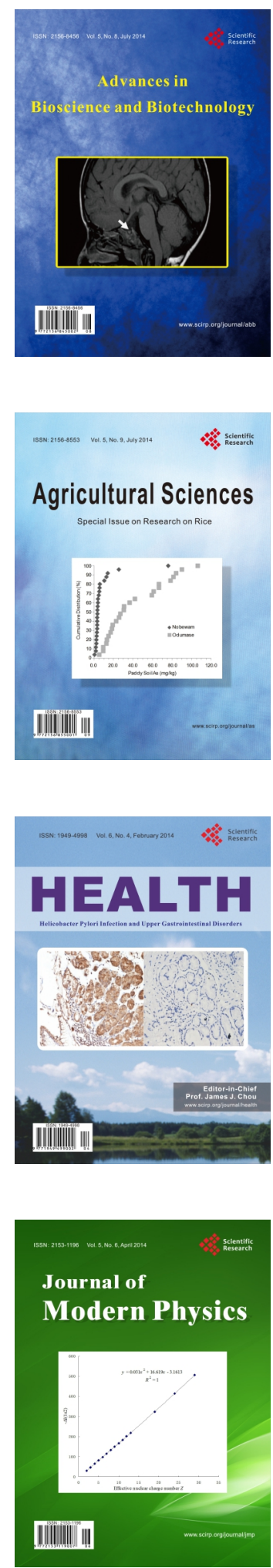
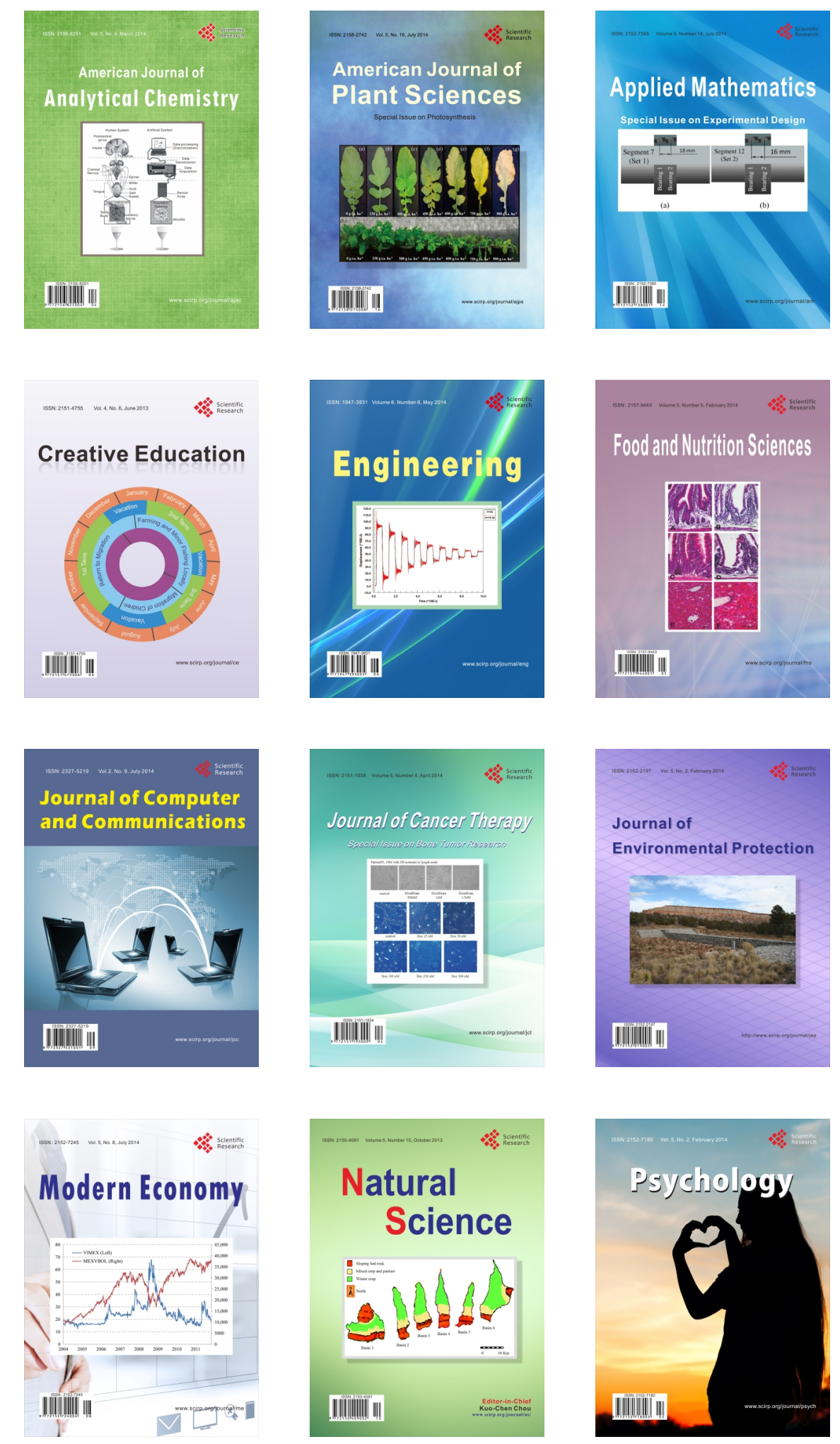